A case of a four month old boy with botulism is reported from the Department of Microbiology, Bristol Royal Infirmary and Bristol Royal Hospital for Sick Children, England. For the week before admission he had profuse rhinorrhea, and for 24 hours there was difficulty in feeding, hypotonia, and respiratory distress. On admission, he was profoundly hypotonic and had bilateral ptosis, impassive facies, depressed deep tendon reflexes and gag reflex, and pharyngeal pooling of saliva. He was mentally alert and responded to painful stimuli. Intravenous edrophonium $(0.1$ $\mathrm{mg} / \mathrm{kg}$ ) had no effect. EMG showed reduced amplitude of motor action potentials. EEG showed generalized high amplitude slow wave activity. Intermittent positive pressure ventilation was required and he began to show improvement by day 18 and was extubated on day 24. Clostridium botulinum and its toxin were isolated from the feces. (Smith GE et al. Infantile botulism. Arch Dis Child June 1989; 64:871-2).

COMMENT. Infantile botulism is rare, this being only the second case in the United Kingdam. The diagnosis should be entertained in infants with an acute onset of hypotonia and respiratory distress. Honey has been implicated in several cases from the United States. (Arnon, SS. Annu Rev Med 1980; 31:541). Fortunately, almost all infants recover completely, the illness lasting between 3 and 20 weeks, but supportive therapy is necessary and the differentiation from neonatal myasthenia gravis, septicemia, and infectious polyneuritis is important.

Botulimum toxin is not all bad! Local intradermal injections of botulinum $A$ toxin may be useful in the treatment of eyelid and facial spasms in patients with generalized dystonias according to a report fram the University of California School of Medicine, San Francisco (Sieff SR. Use of botulinum toxin to treat blepharospasm in a 16 year old with dystonic syndrame. Pediatr Neurol Mar/Apr 1989; $5: 121-3)$. Very small doses of botulinum A toxin are required but the effect is limited to a few months.

\title{
FEVER AND RECURRENT FEBRILE SEIZURES
}

The relation of the height of the fever to the recurrence rate of febrile convulsions was studied in 154 children admitted to the Paediatric Department, Ahmadi Hospital, Kuwait. The children were divided into three groups according to the height of the fever recorded on presentation. Group 1, temperature greater than $40^{\circ} \mathrm{C}$, group $2,39-39.9^{\circ} \mathrm{C}$, and group 3 , $38-38.9^{\circ} \mathrm{C}$. The children were followed at three month intervals for a mean of 40 months (range 2-7) and recurrences occurred between one and 22 months after the original first febrile seizure. The recurrence rates of febrile convulsions were significantly greater in infants aged 6 to 18 months in whom the initial febrile convulsions had been associated with a lower temperature. The rate of recurrence was over nine times higher in infants who had the lowest fever, $38-38.9^{\circ} \mathrm{C}$ and over seven times higher in infants with temperatures of $39-39.9^{\circ} \mathrm{C}$. Repeated convulsions with each febrile episode occurred in only four children (3\%). El-Radhi AS and Banajeh S. Effect of fever on recurrence rate of febrile convulsions. Arch Dis Child June $1989 ; 64: 869-870$ ). 
COMMENT. These findings are confirmation of a febrile seizure threshold dependent on the height of the fever first proposed 30 years ago (Millichap JG. Studies in febrile seizures I. Height of body temperature as a measure of the febrile seizure threshold. Pediatrics $1959 ; 23: 76)$. It is not surprising that children who convulse with a relatively low fever have a poorer prognosis than those convulsing only with high fevers. Another factor noted in many studies to increase the risk of recurrence of febrile seizures is an early age at the first febrile seizure. Frantzen et al (1968) found that children who experienced their first seizure at less than 13 months of age had a 2.3:1 chance for developing further febrile seizures compared to a $1: 2$ chance when the seizure occurred between 14 and 32 months of age and a 1:5 risk when the onset was after 32 months of age. Nelson and Ellenberg (1978) found a $50 \%$ recurrence rate with the onset in the first year of life campared to a $28 \%$ recurrence rate when the onset was after the first year. The height of the fever is a most important determinant of the occurrence of febrile seizures in the infant and young child.

\section{NEURODEGENERATIVE DISORDERS}

\section{NEUROAXONAL DYSTROPHY}

The clinical, pathological and biochemical findings in two brothers with a newly recognized form of infantile neuroaxonal dystrophy associated with alpha-N-acetylgalactosaminidase deficiency are reported from the Divisions of Medical and Molecular Genetics and Neuropathology, Mount Sinai School of Medicine, New York; Department of Chemistry, University of Alberta, Edmonton, Canada; Department of Physiological Chemistry, University of Bonn, Federal Republic of Germany; and Department of Hum Genetics, University of Wurzburg, Federal Republic of Germany. The brothers were the offspring of fifth cousins of German descent and their early development was normal. In the older brother, the clinical onset of disease was signaled by poor coordination of gait, clumsiness, and episodes of falling at 12 months. In the younger brother, grand mal seizures began at eight months and occurred five times over the next six months. Each had a regressive course beginning after 15 months of age, with loss of all mental and motor skills acquired previously. Strabismus, nystagmus, vi sual impairment, muscular hypotonia, and frequent myoclonic movements developed in both. By three to four years of age, both brothers had profound psychomotor retardation and spasticity, and were immobile and incontinent. CT and MRI showed atrophy of the cerebellum, brain stem and cervical spinal cord, with lesser atrophic changes in the cerebral gray and white matter, optic tracts and cranial nerves. EFG revealed multifocal isolated spikes and spike wave complexes. The urinary oligosaccharide profile was abnormal, suggesting the possibility of a lysosomal disease. The activity of alpha-N-acetylgalactosaminidase was deficient whereas 21 other lysosamal enzymes were normal. (Schindler D et al. Neuroaxonal dystrophy due to lysosamal alpha-N-acetylgalactosaminidase deficiency. N Engl J Med June 29,$1989 ; 320: 1735-1740$ ). 